\title{
VENUS
}

\section{Lasting habitability}

J. Geophys. Res. Planets https://doi.

org/10.1029/2019JE006276 (2020)

Even though the image of Venus as Earth's 'evil twin' is a stereotype that is increasingly rejected by planetary scientists, it is now quite well established that the two planets formed in each other's proximity, with the same radius and most probably the same building blocks for composition. The question of when they diverged - that is, for how long Venus was 'Earth-like' or at least potentially habitable - thus arises naturally. Michael Way and Anthony Del Genio show that Venus might have been hospitable to life for most of its history.

Way and Del Genio follow the evolution of Venus across its history by running various simulations within a three-dimensional climate model, with varying initial liquid water abundances, atmospheric pressures and compositions, and solar energy flux. The results show that Venus might have followed the Earth's evolutionary path quite closely after accretion, cooling rapidly and maintaining a temperate climate and surface oceans, for a long time, helped by the carbonate-silicate cycle and volatile recycling. Such a balance would have been thwarted by geological processes, active over a period of tens or hundreds of millions of years, like the onset of widespread volcanism and global resurfacing. These would have warmed Venus to the point of triggering runaway greenhouse, inducing massive water loss and blocking its active tectonics.

The work of Way and Del Genio excludes a progressive early rush towards the present hellish conditions, with important implications for the evolution and habitability of Earth-sized exoplanets, but huge uncertainties still remain on the timing of the described processes, as they might have ended as early as three billion years ago or as late as a few hundred million years ago. Only a new dedicated mission to Venus will be able to provide an answer.

Luca Maltagliati

Published online: 1 May 2020

https://doi.org/10.1038/s41550-020-1110-7 